\title{
On the Stanley depth of squarefree Veronese ideals
}

\author{
Mitchel T. Keller • Yi-Huang Shen • Noah Streib • \\ Stephen J. Young
}

Received: 21 October 2009 / Accepted: 7 July 2010 / Published online: 5 August 2010

(C) The Author(s) 2010. This article is published with open access at Springerlink.com

\begin{abstract}
Let $K$ be a field and $S=K\left[x_{1}, \ldots, x_{n}\right]$. In 1982, Stanley defined what is now called the Stanley depth of an $S$-module $M$, denoted sdepth $(M)$, and conjectured that $\operatorname{depth}(M) \leq \operatorname{sdepth}(M)$ for all finitely generated $S$-modules $M$. This conjecture remains open for most cases. However, Herzog, Vladoiu and Zheng recently proposed a method of attack in the case when $M=I / J$ with $J \subset I$ being monomial $S$-ideals. Specifically, their method associates $M$ with a partially ordered set. In this paper we take advantage of this association by using combinatorial tools to analyze squarefree Veronese ideals in $S$. In particular, if $I_{n, d}$ is the squarefree Veronese ideal generated by all squarefree monomials of degree $d$, we show that if $1 \leq d \leq n<5 d+4$, then $\operatorname{sdepth}\left(I_{n, d}\right)=\lfloor(n-d) /(d+1)\rfloor+d$, and if $d \geq 1$ and $n \geq 5 d+4$, then $d+3 \leq$ $\operatorname{sdepth}\left(I_{n, d}\right) \leq\lfloor(n-d) /(d+1)\rfloor+d$.
\end{abstract}

Keywords Stanley depth · Squarefree monomial ideal · Interval partition · Squarefree Veronese ideal

M.T. Keller $(\bowtie) \cdot$ N. Streib · S.J. Young

School of Mathematics, Georgia Institute of Technology, Atlanta, GA 30332-0160, USA e-mail: keller@math.gatech.edu

N. Streib

e-mail: nstreib3@math.gatech.edu

Y.-H. Shen

Department of Mathematics, University of Science and Technology of China, Hefei, Anhui 230026, China

e-mail: yshen.math@gmail.com

Present address:

S.J. Young

Department of Mathematics, University of California, San Diego, La Jolla, CA 92093-0112, USA

e-mail: s7young@math.ucsd.edu 


\section{Introduction}

Let $K$ be a field and $S=K\left[x_{1}, \ldots, x_{n}\right]$ be the polynomial ring in $n$ variables with coefficients from $K$. The ring $S$ is naturally equipped with a $\mathbb{Z}^{n}$-grading. A Stanley decomposition of a finitely generated $\mathbb{Z}^{n}$-graded $S$-module $M$ is a representation as a finite direct sum of $K$-vector spaces

$$
\mathscr{D}: M=\bigoplus_{i=1}^{m} u_{i} K\left[Z_{i}\right],
$$

where each $u_{i} \in M$ is homogeneous, and $Z_{i}$ is a subset of $\left\{x_{1}, \ldots, x_{n}\right\}$. The Stanley depth of this decomposition is $\operatorname{sdepth}(\mathscr{D}):=\min \left\{\left|Z_{i}\right| \mid 1 \leq i \leq m\right\}$, where $\left|Z_{i}\right|$ is the cardinality of $Z_{i}$. The Stanley depth of $M$ is defined to be

$$
\operatorname{sdepth}(M):=\max \{\operatorname{sdepth}(\mathscr{D}) \mid \mathscr{D} \text { is a Stanley decomposition of } M\} .
$$

In [17], Stanley conjectured that $\operatorname{depth}(M) \leq \operatorname{sdepth}(M)$ for all finitely generated $\mathbb{Z}^{n}$ graded $S$-modules $M$. This conjecture has been confirmed in several special cases, for instance, when the module $M$ is clean in the sense of Dress [8], that is, it allows a homogeneous prime filtration $\mathscr{F}$ with $\operatorname{Supp}(\mathscr{F})=\operatorname{Min}(M)$. The Stanley-Reisner ring $K[\Delta]$ of a simplicial complex $\Delta$ is clean if and only if $\Delta$ is shellable. It was proved in [15] that Stanley's conjecture also holds for $M=S / I$, where $I \subset S$ is a monomial ideal and $\operatorname{dim}(S) \leq 5$. For other recent developments, see for example $[2,3,6,10,11]$.

However, Stanley's conjecture still remains open, partly because of the difficulty of computing the Stanley depth of $M$. Herzog, Vladoiu and Zheng proposed a method of attack in the special case where $M=I / J$ with $J \subset I$ being monomial $S$-ideals in [11]. They associate $I / J$ with a poset $P_{I / J}$, and thus the computation of $\operatorname{sdepth}(I / J)$ is reduced to a corresponding computation on the poset $P_{I / J}$. We briefly review their method for squarefree monomial ideals in Sect. 2. Though a direct implementation of this method is computationally expensive, it has yielded a wealth of information; see $[4,5,12-14,16]$ for its applications.

In this paper, we investigate the squarefree Veronese ideal $I_{n, d}$, which is generated by all squarefree monomials of degree $d$ in $S=K\left[x_{1}, \ldots, x_{n}\right]$. The ideal $I_{n, d}$ is the Stanley-Reisner ideal of the $(d-1)$-skeleton of the $n$-simplex, hence the StanleyReisner ring $S / I_{n, d}$ is Cohen-Macaulay of dimension $n-d$. The ideal $I_{n, d}$ is a polymatroidal ideal [9], and has linear quotients. The Rees algebras of squarefree Veronese ideals and their $a$-invariants were studied in [1].

Herzog et al. showed in [11] that Stanley's conjecture holds for both $I_{n, d}$ and $S / I_{n, d}$. However, it is still difficult to determine $\operatorname{sdepth}\left(I_{n, d}\right)$. The ideal $I_{n, 1}$ is the homogeneous maximal ideal $\left(x_{1}, \ldots, x_{n}\right)$ of $S$. Herzog et al. conjectured in [11] that $\operatorname{sdepth}\left(I_{n, 1}\right)=\left\lceil\frac{n}{2}\right\rceil$. This formula was later confirmed by Biró, Howard, Keller, Trotter and Young in [4] using combinatorial techniques upon which this paper builds. The Stanley depth of $I_{n, d}$ in the other situation remains unexplored in general, although Cimpoeaş considered a similar problem in [7]. In this paper, we prove an exact formula for the Stanley depth of $I_{n, d}$ for certain values of $n$ and $d$ and a bound for others. Our principal result is the following theorem: 
Theorem 1.1 Let $K$ be a field, $S=K\left[x_{1}, \ldots, x_{n}\right]$ be a polynomial ring in $n$ variables over $K$. Furthermore, let $I_{n, d}$ be the squarefree Veronese ideal in $S$, generated by all squarefree monomials of degree $d$.

1. If $1 \leq d \leq n<5 d+4$, then the Stanley depth $\operatorname{sdepth}\left(I_{n, d}\right)=\left\lfloor\frac{n-d}{d+1}\right\rfloor+d$.

2. If $d \geq 1$ and $n \geq 5 d+4$, then

$$
d+3 \leq \operatorname{sdepth}\left(I_{n, d}\right) \leq\left\lfloor\frac{n-d}{d+1}\right\rfloor+d .
$$

We begin the paper by reviewing the method of Herzog et al. for associating a poset with a monomial ideal. We also introduce our notation and prove some preliminary results in Sect. 2. Section 3 is devoted to proving Theorem 1.1 in three stages.

\section{Background, notation, and preliminary results}

\subsection{From ideals to interval partitions}

A monomial in the polynomial ring $K\left[x_{1}, \ldots, x_{n}\right]$ has the form $x_{1}^{i_{1}} \cdots x_{n}^{i_{n}}$ where the $i_{j} \geq 0$ are integers. For each $\mathbf{c}=(c(1), \ldots, c(n)) \in \mathbb{N}^{n}$, denote $\mathbf{x}^{\mathbf{c}}=\prod_{i} x_{i}^{c(i)}$. The monomial $\mathbf{x}^{\mathbf{c}}$ is squarefree precisely when $c(i) \in\{0,1\}$ for $1 \leq i \leq n$. If $\mathbf{x}^{\mathbf{c}}$ is squarefree, let $\operatorname{Supp}\left(\mathbf{x}^{\mathbf{c}}\right)=\{i \mid c(i)=1\} \subseteq[n]:=\{1, \ldots, n\}$ be the support of $\mathbf{x}^{\mathbf{c}}$. A monomial ideal is squarefree if it is generated by squarefree monomials. Thus, the ideal $I_{n, d}$ is squarefree. The method of Herzog et al. [11] for determining the Stanley depth of a squarefree monomial ideal $I$ using posets can be summarized in the following way. Let $G(I)=\left\{v_{1}, \ldots, v_{m}\right\}$ be the unique set of minimal monomial generators of $I$. By definition, each $v_{i}$ is squarefree. For this squarefree ideal $I$, there is a poset with ground set

$$
P_{I}=\left\{C \subseteq[n] \mid C \text { contains } \operatorname{Supp}\left(v_{i}\right) \text { for some } i\right\}
$$

partially ordered by inclusion. The poset $P_{I}$ is a subposet of the Boolean algebra of subsets of $[n]$; it consists of all supersets of the support of the generators of $I$. For every $A, B \in P_{I}$ with $A \subseteq B$, define the interval $[A, B]$ to be $\left\{C \in P_{I} \mid A \subseteq C \subseteq B\right\}$. Let $\mathscr{P}: P_{I}=\bigcup_{i=1}^{r}\left[C_{i}, D_{i}\right]$ be a partition of $P_{I}$, and for each $i$, let $\mathbf{c}_{i} \in \mathbb{N}^{n}$ be such that $\operatorname{Supp}\left(\mathbf{x}^{\mathbf{c}_{i}}\right)=C_{i}$. There is then a Stanley decomposition $\mathscr{D}(\mathscr{P})$ of $I$ :

$$
\mathscr{D}(\mathscr{P}): I=\bigoplus_{i=1}^{r} \mathbf{x}^{\mathbf{c}_{i}} K\left[\left\{x_{j} \mid j \in D_{i}\right\}\right] .
$$

It is clear that $\operatorname{sdepth}(\mathscr{D}(\mathscr{P}))=\min \left\{\left|D_{1}\right|, \ldots,\left|D_{r}\right|\right\}$. Most importantly, Herzog et al. showed in [11] that if $I$ is a squarefree monomial ideal, then

$$
\operatorname{sdepth}(I)=\max \left\{\operatorname{sdepth}(\mathscr{D}(\mathscr{P})) \mid \mathscr{P} \text { is a partition of } P_{I}\right\} .
$$

Before introducing our combinatorial approach to this problem, we establish some bounds on the Stanley depth of squarefree Veronese ideals. We also prove other results which show that our approach to proving Theorem 1.1 can focus on values of $n$ having a particular form. 
Lemma 2.1 If I is a squarefree monomial ideal and $G(I)$ is the minimal monomial generating set of $I$, then $\operatorname{sdepth}(I) \geq \min \{\operatorname{deg} u \mid u \in G(I)\}$.

Proof This follows directly from the method of Herzog, et al. [11] described just prior to this lemma.

Lemma 2.2 For integers $1 \leq d \leq n, \operatorname{sdepth}\left(I_{n, d}\right) \leq\left(\begin{array}{c}n \\ d+1\end{array}\right) /\left(\begin{array}{l}n \\ d\end{array}\right)+d=\frac{n-d}{d+1}+d$.

Proof Since $I_{n, d}$ is generated by monomials of degree $d$, by Lemma 2.1 we have $k:=$ $\operatorname{sdepth}\left(I_{n, d}\right) \geq d$. The poset $P_{I_{n, d}}$ has a partition $\mathscr{P}: P_{I}=\bigcup_{i=1}^{r}\left[C_{i}, D_{i}\right]$, satisfying $\operatorname{sdepth}(\mathscr{D}(\mathscr{P}))=k$. For each interval $\left[C_{i}, D_{i}\right]$ in $\mathscr{P}$ with $\left|C_{i}\right|=d$, we have $\left|D_{i}\right| \geq k$. Furthermore, there are $\left|D_{i}\right|-\left|C_{i}\right|$ subsets of cardinality $d+1$ in this interval. Since these intervals are disjoint, counting the number of subsets of cardinality $d+1$, we have the inequality:

$$
\left(\begin{array}{l}
n \\
d
\end{array}\right)(k-d) \leq\left(\begin{array}{c}
n \\
d+1
\end{array}\right) .
$$

After simplification, we get the desired estimate.

The following corollary is now immediate.

Corollary 2.3 If $d \geq\left\lceil\frac{n}{2}\right\rceil$, then $\operatorname{sdepth}\left(I_{n, d}\right)=d$.

We expect the following conjecture, which generalizes our Theorem 1.1, to be true.

Conjecture 2.4 For positive integers $1 \leq d \leq n$,

$$
\operatorname{sdepth}\left(I_{n, d}\right)=\left\lfloor\left(\begin{array}{c}
n \\
d+1
\end{array}\right) /\left(\begin{array}{l}
n \\
d
\end{array}\right)\right\rfloor+d=\left\lfloor\frac{n-d}{d+1}\right\rfloor+d .
$$

This formula is already known to be valid for $d=1$, see [4]. A further implementation by computer shows that it is also valid for $1 \leq d \leq n \leq 13$.

The structure of the Boolean algebra of subsets of $[n]$ provides the following lemma which allows us to use known bounds on the Stanley depth of a squarefree Veronese ideal to establish other bounds.

Lemma 2.5 Suppose $d, n, a$ are positive integers such that $\operatorname{sdepth}\left(I_{n, d-1}\right) \geq a$ and $\operatorname{sdepth}\left(I_{n, d}\right) \geq a+1$. Then $\operatorname{sdepth}\left(I_{n+1, d}\right) \geq a+1$.

Proof The poset $P_{I_{n, d-1}}$ has a partition $\mathscr{P}_{1}$ with $\operatorname{sdepth}\left(\mathscr{D}\left(\mathscr{P}_{1}\right)\right)=a$, while $P_{I_{n, d}}$ has a partition $\mathscr{P}_{2}$ with $\operatorname{sdepth}\left(\mathscr{D}\left(\mathscr{P}_{2}\right)\right)=a+1$. Now

$$
\mathscr{P}: P_{I_{n+1, d}}=\left(\bigcup_{[C, D] \in \mathscr{P}_{1}}[C \cup\{n+1\}, D \cup\{n+1\}]\right) \cup \mathscr{P}_{2}
$$

is a partition of $P_{I_{n+1, d}}$ with $\operatorname{sdepth}(\mathscr{D}(\mathscr{P}))=a+1$. 
Notice that when $\left(\begin{array}{c}n \\ d+1\end{array}\right) /\left(\begin{array}{l}n \\ d\end{array}\right)=c-1 \in \mathbb{Z}$, we have $n=c d+c-1$. In fact, the following lemma establishes that if we can prove Conjecture 2.4 for such values of $n$, it is true for all $n$ and $d, 1 \leq d \leq n$.

Lemma 2.6 Suppose $\operatorname{sdepth}\left(I_{c d+c-1, d}\right)=d+c-1$ for all positive integers $c$ and $d$. Then $\operatorname{sdepth}\left(I_{n, d}\right) \geq d+c-1$ for all $c$ such that $c d+c-1 \leq n$.

Proof The proof proceeds by double induction, first on $d$ and then on $n$. For the induction on $d$, notice that in [4], Biro et al. proved that when $d=1, \operatorname{sdepth}\left(I_{n, d}\right) \geq$ $\lceil n / 2\rceil$. So if $n \geq c d+c-1=2 c-1$ then

$$
\operatorname{sdepth}\left(I_{n, d}\right) \geq\left\lceil\frac{n}{2}\right\rceil \geq\left\lceil\frac{2 c-1}{2}\right\rceil=c=d+c-1
$$

So suppose that for all $1 \leq d^{\prime} \leq d$ and for all $c \geq 1$, we have for all $n \geq c d^{\prime}+c-1$ that $\operatorname{sdepth}\left(I_{n, d}\right) \geq d^{\prime}+c-1$.

Now consider $I_{n, d+1}$. For this, we use induction on $n$. Since $n \geq c(d+1)+c-1$ and $c \geq 1$, the base case for $n$ is $d+1$. This case follows from the assumption of the lemma with $c=1$. By assumption we have that if $n=c(d+1)+c-1$ for some $c$, then $\operatorname{sdepth}\left(I_{n, d+1}\right) \geq(d+1)+c-1$. Hence, for all $1 \leq c^{\prime} \leq c$ for which $n \geq c^{\prime}(d+1)+c^{\prime}-1$, we have $\operatorname{sdepth}\left(I_{n, d+1}\right) \geq c^{\prime}(d+1)+c^{\prime}-1$.

Thus suppose there exists some $c^{\prime \prime} \geq 1$ such that

$$
c^{\prime \prime}(d+1)+c^{\prime \prime}-1<n<\left(c^{\prime \prime}+1\right)(d+1)+\left(c^{\prime \prime}+1\right)-1 .
$$

As $n-1 \geq c^{\prime \prime}(d+1)+c^{\prime \prime}-1$, our inductive hypothesis on $n$ yields $\operatorname{sdepth}\left(I_{n-1, d+1}\right) \geq(d+1)+c^{\prime \prime}-1$. Also, $n>c^{\prime \prime}(d+1)+c^{\prime \prime}-1=c^{\prime \prime} d+2 c-1$, and therefore we have $n-1>c^{\prime \prime} d+c^{\prime \prime}-1$. Thus the inductive hypothesis for $d$ yields $\operatorname{sdepth}\left(I_{n-1, d}\right) \geq d+c^{\prime \prime}-1$. Thus, by Lemma $2.5, \operatorname{sdepth}\left(I_{n, d+1}\right) \geq(d+1)+c^{\prime \prime}-1$, as desired. This concludes both inductions and finishes the proof of the lemma.

Remark 2.7 Observe that if $c=\left\lfloor\frac{n+1}{d+1}\right\rfloor$ then $c d+c-1 \leq n$. Thus if $\operatorname{sdepth}\left(I_{c d+c-1, d}\right) \geq c+d-1$ for all positive integers $c$ and $d$, then by Lemma 2.2 and Lemma 2.6 we have that for all $n \geq d$,

$$
d+\left\lfloor\frac{n-d}{d+1}\right\rfloor=\left\lfloor\frac{n+1}{d+1}\right\rfloor+d-1 \leq \operatorname{sdepth}\left(I_{n, d}\right) \leq d+\left\lfloor\frac{\left(\begin{array}{c}
n \\
d+1
\end{array}\right)}{\left(\begin{array}{l}
n \\
d
\end{array}\right)}\right\rfloor=d+\left\lfloor\frac{n-d}{d+1}\right\rfloor .
$$

It follows from Remark 2.7 that Conjecture 2.4 can be proved by showing that $\operatorname{sdepth}\left(I_{c d+c-1, d}\right) \geq d+c-1$ for all positive integers $c$ and $d$. In other words, we may restrict our attention to $I_{n, d}$ where $1 \leq d \leq n$ and $n=c d+c-1$ for some $c \geq 1$. Our focus now becomes proving this stronger conjecture, stated below as Conjecture 2.8, for particular values of $c$.

Conjecture 2.8 For positive integers $c$ and $d, \operatorname{sdepth}\left(I_{c d+c-1, d}\right) \geq d+c-1$. 


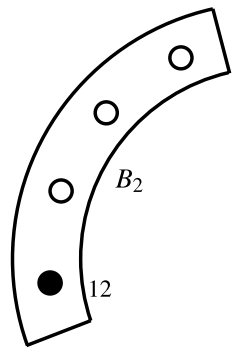

○ $G_{1}$

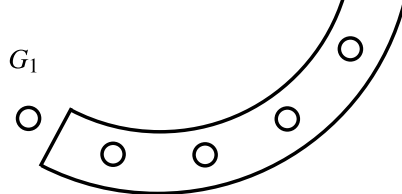

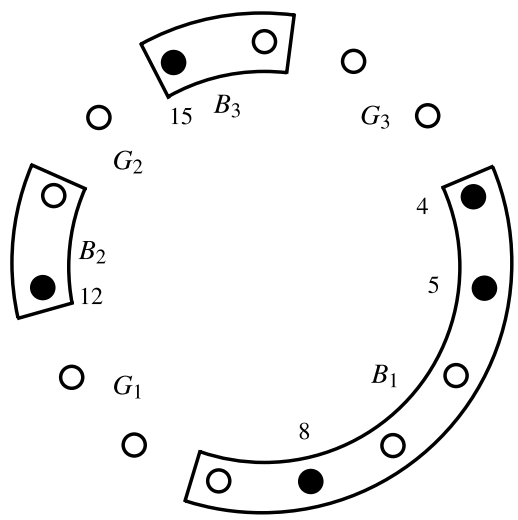

Fig. 1 Two examples of block structures of $A$ with respect to $\delta$. On the left, $A=\{2,4,12\}$ and $\delta=4$. On the right, $A=\{4,5,8,12,15\}$ and $\delta=13 / 6$

\subsection{Combinatorial tools and definitions}

In this paper, we will frequently refer to the Boolean algebra of subsets of $[n]$ partially ordered by inclusion as the $n$-cube. A $t$-set is a subset of $[n]$ of size $t$. Recall that for $A \subseteq B$ in $[n]$, we have defined the interval $[A, B]$ as the set of all $C \subseteq[n]$ such that $A \subseteq C \subseteq B$. Notice that $[A, B]$ is isomorphic to the $(|B|-|A|)$-cube. We say that an interval $[A, B]$ covers a set $C$ if $C \in[A, B]$. For our purposes, it is helpful to think of a subset $S$ of $[n]$ by evenly distributing the points $1,2, \ldots, n$ around a circle in the plane, with the elements of $S$ depicted as solid points and the elements of $[n] \backslash S$ depicted as open points. As such, the circle will have inherent clockwise and counterclockwise directions. We will refer to this as the circular representation of $[n]$.

In order to facilitate our proofs in this paper, we first establish some definitions. Given the circular representation of $[n]$, a block is a subset of consecutive points on the circle. For $i, j \in[n]$ we denote by $[i, j]$ the block starting at $i$ and ending at $j$ when traversing the circular representation of [n] clockwise. Let $A \subseteq[n]$ and let $\delta \in \mathbb{R}$ with $\delta \geq 1$ be a density. The block structure of $A$ with respect to $\delta$ is a partition of the elements of the circular representation of $[n]$ into clockwise-consecutive blocks $B_{1}, G_{1}, B_{2}, G_{2}, \ldots, B_{k}, G_{k}$ such that

(i) the first (going clockwise) element $b_{i}$ of $B_{i}$ is in $A$,

(ii) for all $i \in[k], G_{i} \cap A=\varnothing$,

(iii) for all $i \in[k], \delta \cdot\left|A \cap B_{i}\right|-1<\left|B_{i}\right| \leq \delta \cdot\left|A \cap B_{i}\right|$,

(iv) for all $y \in B_{i}$ such that $\left[b_{i}, y\right] \subsetneq B_{i},\left|\left[b_{i}, y\right]\right|+1 \leq \delta \cdot\left|\left[b_{i}, y\right] \cap A\right|$.

The purpose of (iii) and (iv) in the definition of block structure above is to ensure that the density of elements of $A$ in a block is close to $1 / \delta$. For two examples of a block structure, see Fig. 1.

Lemma 2.9 For $1 \leq \delta \leq(n-1) /|A|$ the block structure for a set $A$ on $[n]$ exists and is unique. 
Proof It is clear for $\delta$ in this range that a block structure can be constructed by iteratively expanding blocks. That is, for each element of $A$ assign the next $\lfloor\delta\rfloor$ elements cyclically to the block containing $A$. If any of these blocks contain another element of $A$, merge the two blocks and then add points from $[n] \backslash A$ if the number of elements of $A$ in the block allows. This process will eventually terminate, yielding a block structure, since $\delta|A| \leq n-1$.

Now suppose that $B_{1}, G_{1}, \ldots, B_{k}, G_{k}$ and $B_{1}^{\prime}, G_{1}^{\prime}, \ldots, B_{k^{\prime}}^{\prime}, G_{k^{\prime}}^{\prime}$ are two block structures for the set $A$ with different blocks. Then there are two blocks, say $B_{i}$ and $B_{j}^{\prime}$, which are not the same and overlap. From (iii) and (iv) it is clear that $b_{i} \neq b_{j}^{\prime}$ and so without loss of generality we may assume that $b_{i}$ precedes $b_{j}^{\prime}$ in the clockwise ordering. We then note that if $B_{j}^{\prime} \backslash B_{i}$ is nonempty; that is, there is an element of $B_{j}^{\prime}$ following $B_{i}$, then

$$
\begin{aligned}
\left|B_{i}\right| & =\left|B_{i} \backslash B_{j}^{\prime}\right|+\left|B_{i} \cap B_{j}^{\prime}\right| \\
& \leq \delta\left|\left(B_{i} \backslash B_{j}^{\prime}\right) \cap A\right|-1+\delta\left|\left(B_{i} \cap B_{j}^{\prime}\right) \cap A\right|-1 \\
& =\delta\left|B_{i} \cap A\right|-2,
\end{aligned}
$$

where the inequality comes from (iv), applied to the initial segments of $B_{i}$ and $B_{j}^{\prime}$, respectively. Note that this contradicts (iii), as $\delta\left|B_{i} \cap A\right|-1$ is not less than $\left|B_{i}\right|$. Furthermore, this implies that $B_{j}^{\prime} \subseteq B_{i} \backslash\left\{b_{i}\right\}$. But since $b_{i} \in A$, there must be some block $B_{t}^{\prime}(i \neq j)$ that contains $b_{i}$. Clearly, $B_{i} \backslash B_{t}^{\prime}$ is nonempty. Specifically, it contains $B_{j}^{\prime}$, and so by the above, $B_{i} \subset B_{t}^{\prime}$. But then $B_{j}^{\prime} \subset B_{i} \subset B_{t}^{\prime}$, contradicting that the block structure is a partition.

Given a block structure, we denote the set of blocks $\left\{B_{1}, B_{2}, \ldots, B_{k}\right\}$ by $\delta$-blocks $(A)$ and the union $B_{1} \cup B_{2} \cup \cdots \cup B_{k}$ by $\mathscr{B}_{\delta}(A)$. Each $G_{i}$ for $i \in[k]$ is called a gap. We denote the set $\left\{G_{1}, G_{2}, \ldots, G_{k}\right\}$ by $\delta$-gaps $(A)$ and the union $G_{1} \cup G_{2} \cup \cdots \cup G_{k}$ by $\mathscr{G}_{\delta}(A)$. Given density $\delta$, let $f_{\delta}$ be the function that maps each $A \subseteq[n]$ with $|A| \leq(n-1) / \delta$ to $A \cup \mathscr{G}_{\delta}(A) \subseteq[n]$. Throughout this paper we will be concerned with intervals of the form $\left[A, f_{\delta}(A)\right]$.

We will also require the use of some graph theoretic notions. We consider only simple, undirected graphs $G=(V, E)$. The neighborhood of a vertex $v$ is the set of all vertices adjacent to $v$ and is denoted $N(v)$. If $S$ is a set of vertices, then $N(S)$ denotes all vertices not in $S$ that are adjacent to a vertex in $S$. A bipartite graph is a graph whose vertex set can be partitioned into sets $V_{1}$ and $V_{2}$ such that all edges of $G$ have one end in $V_{1}$ and the other in $V_{2}$. A matching in a graph is a set of edges in which no two edges in the set have a common end. A complete matching from $V_{1}$ to $V_{2}$ in a bipartite graph $G$ with bipartition $\left(V_{1}, V_{2}\right)$ is a matching containing precisely one edge incident with each vertex in $V_{1}$. A famous result concerning complete matchings is the following theorem due to Hall.

Theorem 2.10 (Hall) Let $G$ be a bipartite graph with bipartition $\left(V_{1}, V_{2}\right)$. Then $G$ has a complete matching from $V_{1}$ to $V_{2}$ if and only if $|N(S)| \geq|S|$ for all $S \subseteq V_{1}$.

In this paper, we will find the following consequence of Hall's Theorem useful. 
Corollary 2.11 Let $G$ be a bipartite graph with bipartition $V(G)=\left(V_{1}, V_{2}\right)$. If each vertex in $V_{1}$ has degree $t$ and $t$ is the maximum degree of $G$, then $G$ has a complete matching from $V_{1}$ to $V_{2}$.

The following lemma provides the first step toward proving Theorem 1.1.

Lemma 2.12 Let $d \geq 1$ be an integer and $n=2 d+1$. Then $\operatorname{sdepth}\left(I_{n, d}\right)=d+1$.

Proof It suffices to show that the $d$-sets in $P_{I_{n, d}}$ can be covered by disjoint intervals $[A, B]$ with $|B|=d+1$. Consider the bipartite graph in which $V_{1}$ consists of the $d$-sets of $[n], V_{2}$ consists of the $(d+1)$-sets, and a $d$-set is adjacent to precisely the $(d+1)$-sets of which it is a subset. Then every vertex has degree $d+1$, so by applying Corollary 2.11 to $V_{1}$, there is a complete matching from $V_{1}$ to $V_{2}$. The edges of this matching then give rise to intervals of the desired form.

Lemma 2.12 is the case of $n=c d+c-1$ with $c=2$. The next section tackles the more challenging cases of $c=3$ and $c=4$.

\section{Proof of Theorem 1.1}

To prove Theorem 1.1, it suffices by Lemma 2.2, Lemma 2.5, Lemma 2.12 and the proof of Lemma 2.6 to show that if $n=c d+c-1$ and $c \in\{3,4\}$, then $\operatorname{sdepth}\left(I_{n, d}\right)=d+c-1$. To do this, we first prove some lemmas about intervals of the form $\left[A, f_{\delta}(A)\right]$. Our first lemma deals with the disjointness of these intervals in certain cases.

Lemma 3.1 Given a positive integer $n$, let $A, A^{\prime} \subseteq[n]$ with $A \neq A^{\prime}$ and $|A|=\left|A^{\prime}\right|$, and let $\delta \in \mathbb{R}$ with $\delta \geq 1$. If $\left|f_{\delta}(A)\right|-|A| \leq \delta-1$, then $\left[A, f_{\delta}(A)\right]$ does not intersect $\left[A^{\prime}, f_{\delta}\left(A^{\prime}\right)\right]$.

Proof Suppose not. Then $\left[A, f_{\delta}(A)\right]$ and $\left[A^{\prime}, f_{\delta}\left(A^{\prime}\right)\right]$ have nontrivial intersection. In particular, $\left(A \cup A^{\prime}\right)$ is the minimal element of $\left[A, f_{\delta}(A)\right] \cap\left[A^{\prime}, f_{\delta}\left(A^{\prime}\right)\right]$. This implies that $a \in A^{\prime} \cup \mathscr{G}_{\delta}\left(A^{\prime}\right)$ for all $a \in A$, and $a^{\prime} \in A \cup \mathscr{G}_{\delta}(A)$ for all $a^{\prime} \in A^{\prime}$. As $A \neq A^{\prime}$, there exists an $x \in A^{\prime} \backslash A$ satisfying $x \in \mathscr{G}_{\delta}(A)$. Let $B_{1}, G_{1}, B_{2}, \ldots, B_{k}, G_{k}$ be as in the definition of the block structure of $A$ and, without loss of generality, suppose $x \in G_{1}$. Note that

$$
\left|\mathscr{G}_{\delta}(A)\right|=\left|f_{\delta}(A)\right|-|A| \leq \delta-1 .
$$

Therefore, the block in $\delta$-blocks $\left(A^{\prime}\right)$ that contains $x$, say $B^{\prime}$, must also contain $b_{2}^{(1)}$, the first element of $B_{2}$. As $b_{2}^{(1)} \in A$ and $b_{2}^{(1)} \notin \mathscr{G}_{\delta}\left(A^{\prime}\right)$ it must be that $b_{2}^{(1)} \in A^{\prime}$. Let $b_{2}^{(2)}$ be the next element of $B_{2} \cap A$ found when proceeding clockwise around the circular representation of $[n]$. Clearly $b_{2}^{(2)} \in B^{\prime}$, so it must be the case that $b_{2}^{(2)} \in A^{\prime}$ as otherwise $b_{2}^{(2)} \notin f_{\delta}\left(A^{\prime}\right)$. Proceeding clockwise in this manner, we find $A \cap B_{2} \subseteq A^{\prime}$ and $B_{2} \subseteq B^{\prime}$. 
Again, using the fact that $\left|\mathscr{G}_{\delta}(A)\right| \leq \delta-1$ and the fact that $x \in B^{\prime}$, we find that $b_{3}^{(1)}$, the first element of $B_{3}$, is also in $B^{\prime}$. Applying the same argument as was applied to $B_{2}$ we find that $A \cap B_{2} \subseteq A^{\prime}$ and $B_{3} \subseteq B^{\prime}$.

Finally, proceeding clockwise and using analogous arguments, we conclude that $A \cap \mathscr{B}_{\delta}(A) \subseteq A^{\prime}$. However, $A \cap \mathscr{B}_{\delta}(A)=A$, contradicting the fact that $A \nsubseteq A^{\prime}$.

We also require the following lemma about the size of $f_{c}(A)$.

Lemma 3.2 Let $c, d$ be positive integers and let $n=c d+c-1$. Let $A \subseteq[n]$ be $a$ d-set. Then $\left|f_{c}(A)\right|=d+c-1$.

Proof Since $c \in \mathbb{N}$, it follows from the definition of the block structure of $A$ with respect to the density $c$ that $\left|\mathscr{B}_{c}(A)\right|=c|A|$. Since $A$ is a $d$-set, we find that $\left|\mathscr{G}_{c}(A)\right|=$ $n-c d=c-1$. Thus, $\left|f_{c}(A)\right|=|A|+\left|\mathscr{G}_{c}(A)\right|=d+c-1$.

We now show that the intervals of the form $\left[A, f_{c}(A)\right]$ with $|A|=d$ cover all the $(d+1)$-subsets of $[n]$.

Lemma 3.3 Let $c, d$ be positive integers and let $n=c d+c-1$. Let

$$
\mathscr{I}=\left\{\left[A, f_{c}(A)\right]|A \subseteq[n],| A \mid=d\right\} .
$$

Then each $(d+1)$-subset of $[n]$ is covered by a unique element of $\mathscr{I}$.

Proof Lemma 3.2 implies that $\left|f_{c}(A)\right|-|A|=c-1$. Thus, we may apply Lemma 3.1, which implies that each $(d+1)$-set is covered by at most one element of $\mathscr{I}$. So it suffices to show that the number of $(d+1)$-sets covered by the elements of $\mathscr{I}$ is precisely $\left(\begin{array}{c}n \\ d+1\end{array}\right)$.

By Lemma 3.2, each element of $\mathscr{I}$ is a $(c-1)$-cube. Thus, each element of $\mathscr{I}$ covers exactly $c-1$ sets of size $(d+1)$. Since $|\mathscr{I}|=\left(\begin{array}{l}n \\ d\end{array}\right)$, we find that the elements of $\mathscr{I}$ cover $(c-1)\left(\begin{array}{l}n \\ d\end{array}\right)$ sets. Moreover, since $n=c d+c-1$, it is easy to verify that

$$
(c-1)\left(\begin{array}{l}
n \\
d
\end{array}\right)=\left(\begin{array}{c}
n \\
d+1
\end{array}\right)
$$

as desired.

The following proposition resolves the first of our two remaining cases, that of $c=3$.

Proposition 3.4 Let $d$ be a positive integer and let $n=3 d+2$. Then there exists a partition of the subsets of $[n]$ of size at least $d$ into intervals $[A, B]$ such that $|B| \geq d+2$.

Proof Let $\mathscr{I}=\left\{\left[A, f_{3}(A)\right]|A \subseteq[n]| A \mid,=d\right\}$. By Lemma 3.2, $\left|f_{3}(A)\right|=d+2$ for all $d$-sets $A$. By Lemma 3.3, all $(d+1)$-sets are covered by the elements of $\mathscr{I}$. Since Lemma 3.2 implies that $\left|f_{3}(A)\right|-|A|=2$, we can apply Lemma 3.1 to the 
$d$-sets to show that $I \cap I^{\prime}=\varnothing$ for all $I \neq I^{\prime} \in \mathscr{I}$. Moreover, the $(d+2)$-sets that are not covered by any element of $\mathscr{I}$ and the subsets of $[n]$ of size at least $d+3$ can be covered with trivial intervals. Therefore we have found a partition of the desired type.

The next lemma provides the final tool required for our proof. It shows that after selecting the intervals of the form $\left[A, f_{c}(A)\right]$ with $|A|=d$ for our partition, any uncovered set has all of its supersets uncovered as well.

Lemma 3.5 Let $c, d$ be positive integers with $c \geq 2$. Let $n=c d+c-1$ and let $k$ be a positive integer. Let $\mathscr{I}=\left\{\left[A, f_{c}(A)\right]|A \subseteq[n]| A \mid,=d\right\}$. Suppose $D_{k}$ is a $(d+k)$ set that is not covered by any element of $\mathscr{I}$. Then there is no superset of $D_{k}$ that is covered by an element of $\mathscr{I}$.

Proof Suppose not. Let $D^{\prime}$ be a superset of $D_{k}$ that is covered by some $I \in \mathscr{I}$. By Lemma 3.2, I covers some $(d+c-1)$-set, say $D^{\prime \prime}$, that is also a superset of $D_{k}$. Thus, it suffices to prove that there is no superset of $D_{k}$ of size $(d+c-1)$ that is covered by an element of $\mathscr{I}$. To this end, let $\left[D_{0}, f_{c}\left(D_{0}\right)\right] \in \mathscr{I}$ with $D_{k} \subseteq f_{c}\left(D_{0}\right)$. Let $X=f_{c}\left(D_{0}\right) \backslash D_{k}$. We call such a combination of sets $\left(X, D_{0}\right)$ a pair. We call the pair $\left(X, D_{0}\right)$ optimal if, among all pairs, $\left|X \cap D_{0}\right|$ is minimized.

Let $\left(X^{(0)}, D_{0}^{(0)}\right)$ be an optimal pair. Notice that if $X^{(0)} \cap D_{0}^{(0)}=\varnothing$, then since we know $D_{0}^{(0)} \subseteq X^{(0)} \cup D_{k}$, we have that $D_{0}^{(0)} \subseteq D_{k} \subseteq f_{c}\left(D_{0}^{(0)}\right)$. In this case, $\left[D_{0}^{(0)}, f_{c}\left(D_{0}^{(0)}\right)\right]$ covers $D_{k}$, a contradiction. Thus $\left|X^{(0)} \cap D_{0}^{(0)}\right| \geq 1$. Consider $x_{0} \in$ $X^{(0)} \cap D_{0}^{(0)}$. Let $B \in c$-blocks $\left(D_{0}^{(0)}\right)$ such that $x_{0} \in B$. Let $x_{1}$ be the first element in $\mathscr{G}_{c}\left(D_{0}^{(0)}\right)$ counterclockwise from $B$ in the circular representation of [n], and let $z_{0}$ be the last element of $B$ (that is, the largest element in the clockwise order of $B$ ). The point $z_{0}$ exists and is distinct from $x$ since for $\delta \geq 2$, a block cannot end in an element of $A$. Let $x_{2}, \ldots, x_{t}$ be the successive elements of the gaps of $D_{0}^{(0)}$, indexed counterclockwise from $x_{1}$. Fix $s$ as small as possible so that $x_{s+1} \in D_{k}$. Such an $s$ must exist, as otherwise all the gap points belong to $X^{(0)}$, so we would have $\left|X^{(0)}\right| \geq c-1$, but $\left|X^{(0)}\right|=d+c-1-(d+k)=c-1-k<c-1$.

We now define a sequence of pairs $\left(X^{(i)}, D_{0}^{(i)}\right)$. Let $0 \leq i \leq s$ and then define $D_{0}^{(i+1)}=\left(D_{0}^{(i)} \backslash\left\{x_{i}\right\}\right) \cup\left\{x_{i+1}\right\}$. Notice that $f_{c}\left(D_{0}^{(i+1)}\right)=\left(f_{c}\left(D_{0}\right) \backslash\left\{x_{i}\right\}\right) \cup\left\{z_{i}\right\}$, where $z_{i}$ is the last element of the block of $c$-blocks $\left(D_{0}^{(i)}\right)$ that contains $x_{i}$. We then define $X^{(i+1)}=f_{c}\left(D_{0}^{(i+1)}\right) \backslash D_{k}=\left(X^{(i)} \backslash\left\{x_{i}\right\}\right) \cup\left\{z_{i}\right\}$. See, for example, Fig. 2. Now note that we have the following property from our definition.

$$
\begin{aligned}
\left|X^{(i+1)} \cap D_{0}^{(i+1)}\right| & =\left|\left(\left(D_{0}^{(i)} \backslash\left\{x_{i}\right\}\right) \cup\left\{x_{i+1}\right\}\right) \cap\left(\left(X^{(i)} \backslash\left\{x_{i}\right\}\right) \cup\left\{z_{i}\right\}\right)\right| \\
& =\left|\left(\left(D_{0}^{(i)} \cap X^{(i)}\right) \backslash\left\{x_{i}\right\}\right) \cup\left(D_{0}^{(i)} \cap\left\{z_{i}\right\}\right) \cup\left(X^{(i)} \cap\left\{x_{i+1}\right\}\right)\right| \\
& =\left|D_{0}^{(i)} \cap X^{(i)}\right|-1+0+\left|X^{(i)} \cap\left\{x_{i+1}\right\}\right| .
\end{aligned}
$$

For $0 \leq i<s$, we know that $x_{i+1} \notin D_{k}$, and therefore $x_{i+1} \in X^{(i)}$. From the computation above, we see that this implies $\left|X^{(i+1)} \cap D_{0}^{(i+1)}\right|=\left|X^{(i)} \cap D_{0}^{(i)}\right|$. Therefore, 

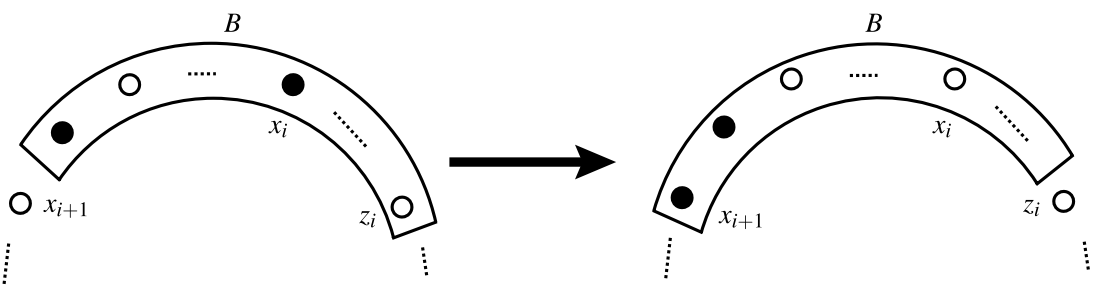

Fig. 2 On the left is the block structure of $D_{0}^{(i)}$, while on the right is the block structure of $D_{0}^{(i+1)}$

the pair $\left(X^{(i+1)}, D_{0}^{(i+1)}\right)$ is optimal for $i<s$. On the other hand, $x_{s+1} \in D_{k}$, and therefore $x_{s+1} \notin X^{(i)}$, so we have $\left|X^{(s+1)} \cap D_{0}^{(s+1)}\right|<\left|X^{(0)} \cap D_{0}^{(0)}\right|$, contrary to the optimality of $\left(X^{(0)}, D_{0}^{(0)}\right)$. Therefore, a set $D_{k}$ as described above cannot exist.

With this result in hand, we are now prepared to resolve the case $c=4$.

Theorem 3.6 Let $d$ be a positive integer, and let $n=4 d+3$. Then there exists a partition of the subsets of $[n]$ of size at least $d$ into intervals $[A, B]$ such that $|B| \geq$ $d+3$.

Proof Let $\mathscr{I}=\left\{\left[A, f_{4}(A)\right]|A \subseteq[n]| A \mid,=d\right\}$. By Lemma 3.2, $\left|f_{4}(A)\right|=d+3$ for all $d$-sets $A$. By Lemma 3.3, all $(d+1)$-sets are covered by the elements of $\mathscr{I}$. Since Lemma 3.2 implies that $\left|f_{4}(A)\right|-|A|=3$, we can apply Lemma 3.1 to the $d$-sets to show that $I \cap I^{\prime}=\varnothing$ for all $I \neq I^{\prime} \in \mathscr{I}$. Therefore we have partitioned all of the $d$-sets and $(d+1)$-sets into the appropriate intervals. What remains to show is that the $(d+2)$-sets and $(d+3)$-sets that are not covered by any element of $\mathscr{I}$ can be partitioned according to the theorem.

For $i \in\{1,2\}$ let $\mathscr{V}_{i}=\left\{S \subseteq[n]|| S \mid=d+i+1, S \notin \bigcup_{[A, B] \in \mathscr{I}}[A, B]\right\}$. Let $G$ be the bipartite graph with vertex set $\mathscr{V}_{1} \cup \mathscr{V}_{2}$ and $S_{1} S_{2} \in E(G)$ if and only if $S_{1} \in \mathscr{V}_{1}$ is a subset of $S_{2} \in \mathscr{V}_{2}$. By Lemma 3.5 with $k=2$ we have that

$$
\operatorname{deg}(S)=n-(d+2) \geq d+3
$$

for each $S \in \mathscr{V}_{1}$. Furthermore, $\operatorname{deg}(S) \leq d+3$ for all $S \in \mathscr{V} / 2$. Hence, by Corollary 2.11 , there exists a complete matching from $\mathscr{V}_{1}$ to $\mathscr{V}_{2}$. Therefore we can use the 1 -cubes corresponding to the matching to cover the elements of $\mathscr{V}_{1}$, and these intervals are disjoint. The remaining elements of $\mathscr{V} / 2$ can then be covered by trivial intervals. Furthermore, the sets of size at least $d+3$ can be covered by trivial intervals as well. Thus, we have found a partition of the desired type.

This completes the proof of Theorem 1.1.

\section{Conclusion}

Although this paper only resolves a fraction of the possible cases of Conjecture 2.4, we believe that it is true in full generality, in part because many of the lemmas in this 
paper are stated and proved in greater generality than is required for their applications here. However, it is not clear how to apply them to advance this line of research. It seems that after applying Lemma 3.5 it may be beneficial to pass from our combinatorial setting back to the algebraic setting to acquire some insight. Although it is not immediately obvious, our approach here generalizes the constructive proof for $d=1$ given by Biró et al. in [4]. The generalization comes through the notion of balanced sets as originally defined there and an equivalent method of finding the intervals. Unfortunately, we do not see how to generalize the method of finding intervals that appears in [4] to higher values of $d$. We also note that the inductive proof for $d=1$ given by Biró et al. seems to face significant challenges in generalizing, and while we would not rule out the use of such a method to prove Conjecture 2.4, it seems unlikely to be successful.

Acknowledgements The authors are grateful to Jürgen Herzog, David M. Howard and William T. Trotter for useful conversations while pursuing this research. Yi-Huang Shen was supported in part by the Fundamental Research Funds for the Central Universities.

Open Access This article is distributed under the terms of the Creative Commons Attribution Noncommercial License which permits any noncommercial use, distribution, and reproduction in any medium, provided the original author(s) and source are credited.

\section{References}

1. Alcántar, A.: Rees algebras of square-free Veronese ideals and their $a$-invariants. Discrete Math. 302, 7-21 (2005)

2. Apel, J.: On a conjecture of R.P. Stanley. I. Monomial ideals. J. Algebr. Comb. 17(1), 39-56 (2003)

3. Apel, J.: On a conjecture of R.P. Stanley. II. Quotients modulo monomial ideals. J. Algebr. Comb. 17(1), 57-74 (2003)

4. Biró, Cs., Howard, D.M., Keller, M.T., Trotter, W.T., Young, S.J.: Interval partitions and Stanley depth. J. Comb. Theory Ser. A. 117(4), 475-482 (2010)

5. Cimpoeaş, M.: Stanley depth of complete intersection monomial ideals. Bull. Math. Soc. Sci. Math. Roum. (N.S.) 51(99)(3), 205-211 (2008)

6. Cimpoeaş, M.: A note on Stanley's conjecture for monomial ideals. arXiv:0906.1303 [math.AC] (2009)

7. Cimpoeaş, M.: Stanley depth of square free Veronese ideals. arXiv:0907.1232 [math.AC] (2009)

8. Dress, A.: A new algebraic criterion for shellability. Beitr. Algebra Geom. 34(1), 45-55 (1993)

9. Herzog, J., Hibi, T.: Cohen-Macaulay polymatroidal ideals. Eur. J. Comb. 27, 513-517 (2006)

10. Herzog, J., Jahan, A.S., Yassemi, S.: Stanley decompositions and partitionable simplicial complexes. J. Algebr. Comb. 27, 113-125 (2008)

11. Herzog, J., Vladoiu, M., Zheng, X.: How to compute the Stanley depth of a monomial ideal. J. Algebra 322(9), 3151-3169 (2009)

12. Keller, M.T., Young, S.J.: Stanley depth of squarefree monomial ideals. J. Algebra 322(10), 37893792 (2009)

13. Nasir, S.: Stanley decompositions and localization. Bull. Math. Soc. Sci. Math. Roum. (N.S.) 51(99)(2), 151-158 (2008)

14. Okazaki, R.: A lower bound of Stanley depth of monomial ideals. J. Commut. Algebra (2009, to appear)

15. Popescu, D.: Stanley depth of multigraded modules. J. Algebra 321(10), 2782-2797 (2009)

16. Shen, Y.H.: Stanley depth of complete intersection monomial ideals and upper-discrete partitions. J. Algebra 321(4), 1285-1292 (2009)

17. Stanley, R.P.: Linear Diophantine equations and local cohomology. Invent. Math. 68, 175-193 (1982) 